\title{
Soluble CCR2 Gene Therapy Controls Joint Inflammation, Cartilage Damage, and the Progression of Osteoarthritis by Targeting MCP-1
}

Hyun Sik Na

The Catholic University of Korea

Seon-Yeong Lee

The Catholic University of Korea

Dong Hwan Lee

Uijeongbu Saint Mary's Hospital

Jin Seok Woo

The Catholic University of Korea

Keun-Hyung Cho

The Catholic University of Korea

Seon Ae Kim

Uijeongbu Saint Mary's Hospital

Eun Jeong Go

Uijeongbu Saint Mary's Hospital

A Ram Lee

The Catholic University of Korea

Jeong-Won Choi

The Catholic University of Korea

Seok Jung Kim

Uijeongbu Saint Mary's Hospital

Mi-La Cho ( $\square$ iammila@catholic.ac.kr)

Catholic University of Korea https://orcid.org/0000-0001-5715-3989

\section{Research Article}

Keywords: CCR2 gene therapy, joint inflammation, cartilage damage, osteoarthritis, MCP-1, sCCR2 E3

Posted Date: September 21st, 2021

DOI: https://doi.org/10.21203/rs.3.rs-886066/v1 
License: (c) (i) This work is licensed under a Creative Commons Attribution 4.0 International License. Read Full License 


\section{Abstract}

Background: Osteoarthritis (OA) is the most common type of degenerative arthritis and affects the entire joint, causing pain, joint inflammation, and cartilage damage. Various risk factors are implicated in causing $\mathrm{OA}$, and in recent years, a lot of research and interest have been directed toward chronic lowgrade inflammation in OA. Monocyte chemoattractant protein-1 (MCP-1; also called CCL2) acts through $\mathrm{C}-\mathrm{C}$ chemokine receptor type 2 (CCR2) in monocytes and is a chemotactic factor of monocytes that plays an important role in the initiation of inflammation. The targeting of CCL2-CCR2 is being studied as part of various topics including the treatment of $O A$.

Methods: In this study, we evaluated the potential therapeutic effects the SCCR2 E3 gene may exert on OA. The effects of SCCR2 E3 were investigated in animal experiments consisting of intra-articular injection of SCCR2 E3 in a monosodium iodoacetate (MIA)-induced OA rat model. The effects after intraarticular injection of SCCR2 E3 (fusion protein encoding 20 amino acids of the E3 domain of the CCL2 receptor) in a monosodium iodoacetate-induced OA rat model were compared to those in rats treated with empty vector (mock treatment) and full-length sCCR2.

Results: Pain improved with expression of the sCCR2 gene. Improved bone resorption upon SCCR2 E3 gene activation was confirmed via bone analyses using micro-computed tomography. Histologic analyses showed that the SCCR2 E3 gene exerted protective effects against cartilage damage and antiinflammatory effects on joints and the intestine.

Conclusions: These results show that SCCR2 E3 therapy is effective in reducing pain severity, inhibiting cartilage destruction, and suppressing intestinal damage and inflammation. Thus, sCCR2 E3 may be a potential therapy for OA.

\section{Background}

Osteoarthritis $(\mathrm{OA})$ is the most common joint disorder in modern society, especially as the number of elderly patients continues to increase; it affects the entire joint with cartilage destruction at its core[1]. As a multifactorial disease, there are various risk factors associated with OA, such as age, obesity, alignment, and mechanical loading, and in recent years, many studies and much interest have been directed toward chronic low-grade inflammation in $O A[1,2]$. As interest in the treatment of early $O A$ is gradually increasing, therapeutic drugs are being researched for use in disease-modifying treatments, and accordingly, many different approaches to OA treatment are being applied[3]. This includes research into the role of cytokines and chemokines in the pathological process of OA, as well as related therapeutic drugs[4].

Monocyte chemoattractant protein-1 (MCP-1/CCL2) is a member of the C-C chemokine family and acts as a chemotactic factor for monocytes[5]. It also plays an important role in the initiation of inflammation through C-C chemokine receptor type 2 (CCR2) in monocytes[6]. Research into which therapeutic effects are obtained by blocking inflammation and tissue damage through CCL2-CCR2 is being conducted for 
various diseases, such as rheumatoid arthritis (RA), cancer, atherosclerosis, myocardial infarction, and $\mathrm{OA}[7-10]$. Inflammation is induced, and cartilage damage occurs through CCL2-CCR2, which plays an important role in OA progression[11, 12].

To block the pathway through CCL2-CCR2 in RA, a method for targeting and blocking CCL2 and CCR2 has been studied. Many clinical trials have been conducted on monoclonal antibodies and smallmolecule drugs, but these have not exhibited efficacy in treating RA [13]. As effective results have not been obtained for other diseases including RA, research on methods based on targeting the CCL2-CCR2 pathway is ongoing[9]. Izhak et al. (2009) showed that a fusion protein comprising 20 amino acids of the third extracellular (E3) domain of the CCL2 receptor and a soluble CCR2 (SCCR2) receptor selectively binds to CCL2 and CCL16, and its binding affinity is effective compared to the $\mathrm{N}$-terminal region or a combination of the $\mathrm{E} 3$ domain and $\mathrm{N}$-terminal region.

In this study, we evaluated the potential therapeutic effects the SCCR2 E3 gene may exert on OA. The effects of SCCR2 E3 were investigated in animal experiments consisting of intra-articular injection of sCCR2 E3 in a monosodium iodoacetate (MIA)-induced OA rat model. The anti-nociceptive effects were analyzed, and the anti-inflammatory effects were assessed by sampling the knee joint and small intestine. Through histological and immunohistochemical (IHC) analyses, the degree of damage to the cartilage and small intestine was evaluated, and the levels of pro-inflammatory cytokines and catabolic factors were determined.

\section{Methods}

Animals. Seven-week-old male Wistar rats weighing 180-250 g each were purchased from Central Lab Animal Inc. (Seoul, South Korea). A maximum of three animals was housed per cage in a room with controlled temperature $\left(20-26^{\circ} \mathrm{C}\right)$ and light (12-h light/dark cycle) conditions. The rats had free access to a gamma ray-sterilized diet (TD 2018S; Harlan Laboratories, Indianapolis, IN, USA) and autoclaved reverse osmosis water. All animal research procedures were conducted in accordance with the Laboratory Animals Welfare Act, the Guide for the Care and Use of Laboratory Animals, and the Guidelines and Policies for Rodent Experiments provided by the Institutional Animal Care and Use Committee (IACUC) of the School of Medicine, the Catholic University of Korea. The IACUC and Department of Laboratory Animals of the Catholic University of Korea, Songeui Campus accredited the Korean Excellence Animal Laboratory Facility in accordance with guidelines of the Korean Food and Drug Administration in 2017, and full accreditation by AAALAC International was achieved in 2018.

\section{Plasmid vector construction}

The rat SCCR2 E3 DNA and rat SCCR2 full DNA were amplification using PCR and the following forward and reverse primers were used: for rat sCCR2 E3, 5'-GTA CGA AGC TTG ACC ACC TTC CAG GAA TTC TTG GGA ATG AGT AAC TGT GTG GTT GAC ATG CAC TTA-3' and 5'-GGT TCC TGC AGG GCC TGG TCT AAG TGC ATG TCA ACC ACA CAG TTA CTC ATT CCC-3'; for rat sCCR2 full, 5'-GGG GAA GCT TAT GGA AGA CAG TAA TAT GTT ACC TC-3' and 5'-GGG ACT GCA GCA ACC CAA CTG AGA CTT CTT GCT C-3'. For 
stability and activity of E3, E3 was conjugated with Fc, and the rat SCCR2 E3-Fc and the rat SCCR2 full were inserted into the pSecTag2A vector.

\section{Transfection of Mock and sCCR2-E3}

Transfection of mock and SCCR2-E3 DNA vector were performed using the DNA transfection reagent (ROCHE X-treameGENE ${ }^{\text {TM }}$; ROCHE, NJ, USA) in OA MSC. After transfection, the OA MSC was stimulated with $100 \mathrm{ng} / \mathrm{mL}$ anti-CD3 and $10 \mathrm{ng} / \mathrm{mL}$ MCP-1 for 12 hours.

Induction of OA and treatment using the SCCR2 gene. Animals were randomly assigned to treatment or control groups before the study began. After anesthetization with isoflurane, the rats were injected with 3 mg of MIA (Sigma, St. Louis, MO, USA) using a $26.5 \mathrm{G}$ needle inserted through the patellar ligament into the intra-articular space of the right knee. The intact knee joint was injected with empty vector (Mock), copies of the sCCR2 E3 gene, or copies of the full-length sCCR2 gene $(100 \mu \mathrm{g} / \mathrm{rat})$.

Assessment of pain behavior. Nociception in MIA-treated rats randomized to the different experimental groups was tested using a dynamic plantar aesthesiometer (Ugo Basile, Gemonio, Italy). The device is an automated version of the von Frey hair assessment system and is used to assess mechanical sensitivity. The assessment was conducted by placing each rat on a metal mesh surface in an acrylic chamber in a temperature-controlled room $\left(20-26^{\circ} \mathrm{C}\right)$, where it rested for $10 \mathrm{~min}$ before the touch stimulator unit was positioned under the animal. An adjustable angled mirror was used to place the stimulating microfilament ( $0.5 \mathrm{~mm}$ diameter) below the plantar surface of the hind paw. When the instrument was activated, a fine plastic monofilament advanced at a constant speed and touched the paw in the proximal metatarsal region. The filament exerted a gradually increasing force on the plantar surface, starting below the threshold of detection and increasing until the stimulus became painful, as indicated by withdrawal of the rat's paw. The force required to elicit a paw-withdrawal reflex was recorded automatically and measured in grams. A maximum force of $50 \mathrm{~g}$ and a ramp speed of $25 \mathrm{~s}$ were used for all aesthesiometer tests.

Assessment of weight bearing. Weight balance in MIA-treated rats was analyzed using an incapacitance meter (IITC Life Sciences, Woodland Hills, CA, USA). The rats were each allowed to acclimate for 5 min in an acrylic holder. Then, two feet were fixed to the pad, and the weight balance was measured for $5 \mathrm{~s}$. Three measurements were repeated in the same manner. The weights on the unguided and guided legs were determined. The percentage weight balance was obtained by comparing legs with and without OA.

Histopathological analyses. Knee joint and intestine samples were collected from each group at 2 weeks post-MIA induction. The tissues were fixed in $10 \%$ formalin solution, decalcified using Decalcifying Solution-Lite (Sigma), and embedded in paraffin. Sections of 4- to 5- $\mu \mathrm{m}$ thickness were cut, dewaxed using xylene, dehydrated through an alcohol gradient, and stained with hematoxylin and eosin (H\&E) and safranin 0 . 
IHC analyses. Paraffin-embedded sections were incubated at $4^{\circ} \mathrm{C}$ with the following primary polyclonal antibodies: anti-interleukin (IL) 1 beta (IL-1 $\beta$ ), anti-IL-6, anti-matrix metalloproteinase 13 (MMP-13) (Abcam, Cambridge, UK), anti-MCP-1, anti-CCR2 (Novus Biologicals, Littleton, CO, USA) and anti-IL-17. Then, the samples were incubated with the respective secondary biotinylated antibodies, followed by 30 min incubation with streptavidin-peroxidase complex. Reaction products were developed using 3, 3diaminobenzidine chromogen (Dako, Carpinteria, CA, USA).

In vivo micro-computed tomography imaging and analyses. Micro-computed tomography (CT) imaging and analyses were performed using a bench-top cone-beam-type in vivo animal scanner (mCT 35; Scanco Medical, Brüttisellen, Switzerland). The animals were imaged at settings of $70 \mathrm{kVp}$ and $141 \mu \mathrm{A}$ using a 0.5 -mm-thick aluminum filter. The pixel size was $8.0 \mu \mathrm{m}$ and the rotation step was $0.4^{\circ}$. Cross-sectional images were reconstructed using a filtered back-projection algorithm (NRecon software; Bruker microCT, Kontich, Belgium). For each scan, a stack of 286 crosssections was reconstructed at 2,000 × 1,335 pixels. The femur bone volume and surface were analyzed.

Real-time PCR. Total RNA isolated from human chondrocytes using TRIzol reagent (Molecular Research Center, Cincinnati, OH, USA) was used to synthesize cDNA. The relative expression of specific mRNAs was quantified via real-time PCR using Sensil FAST SYBR (Bioline, Taunton, MA, USA), and the following sense and antisense primers were used: SRY-box transcription factor 9 (SOX9), 5'-ACT TGC ACA ACG CCG AG-3' (sense) and 5'-CTG GTA CTT GTA ATC CGG GTG-3' (antisense); MMP-1, 5'-CTG AAG GTG ATG AAG CAG CC-3' (sense) and 5'-AGT CCA AGA GAA TGG CCG AG-3' (antisense); MMP-3, 5'-CTC ACA GAC CTG ACT CGG TT-3' (sense) and 5'-CAC GCC TGA AGG AAG AGA TG-3' (antisense).

Statistical analyses. The results are expressed as the mean \pm standard deviation, which were obtained from three separate experiments. Statistical significance was determined via the Mann-Whitney U-test or analysis of variance with Bonferroni's post-hoc test using GraphPad Prism (version 5.01; GraphPad Software, San Diego, CA, USA). $\mathrm{P}<0.05$ was considered to indicate statistical significance.

\section{Results}

SCCR2 regulates the pain threshold in MIA-induced OA rats. To address the role of the sCCR2 gene in vivo (Fig. 1A), we injected copies of full-length sCCR2 or sCCR2 E3 into MIA-induced OA rats (Fig. 1B). Then, we measured paw withdrawal latency (PWL) and determined the paw withdrawal threshold (PWT) using a dynamic plantar aesthesiometer and compared outcomes between the $\mathrm{OA}$ and normal phenotypes. Both PWL and the PWT increased in both SCCR2 groups compared with the empty vector Mock group (Fig. 1C). Also, the weight bearing ability improved in both the full-length SCCR2 and SCCR2 E3 groups compared with the Mock group (Fig. 1D). These results indicate that both the full-length SCCR2 and sCCR2 E3 genes have similar effects in terms of controlling pain.

SCCR2 reduces bone resorption in MIA-induced OA rats. We investigated the role of the SCCR2 gene in bone resorption by performing bone analyses using micro-CT imaging of samples from three groups (Mock, sCCR2 E3, and full-length sCCR2) (Fig. 2A). The bone surface, bone volume, and St. Th were 
increased in the sCCR2 E3 group, indicating less bone loss compared with the Mock and full-length sCCR2 groups (Fig. 2B). These results indicate that the SCCR2 E3 gene exerts protective effects against bone loss.

sCCR2 reduces cartilage damage in MIA-induced OA rats. Knee joint tissue was collected from the Mock, SCCR2 E3, and full-length sCCR2 groups, and safranin O staining was performed. Both the sCCR2 E3 and full-length sCCR2 groups exhibited less proteoglycan depletion, with the SCCR2 E3 group exhibiting less proteoglycan depletion than the full-length SCCR2 group (Fig. 3A). The results of safranin 0 staining were evaluated using the Osteoarthritis Research Society International (OARSI) score and Mankin score for each group. The scores were lower in both the sCCR2 E3 and full-length sCCR2 groups than in the Mock group, and the sCCR2 E3 group had a lower score than the full-length sCCR2 group (Fig. 3B). These results suggest that SCCR2 E3 has a greater protective effect against cartilage destruction than full-length SCCR2.

sCCR2 reduces inflammatory cytokines in the knee joint. IHC staining was performed on the knee joint tissues collected from the Mock, sCCR2 E3, full-length sCCR2 groups. Specific antibodies were used to analyze inflammatory cytokines IL-1 $\beta$ and IL-6, and catabolic factor MMP-13 (Fig. 4A). There were less IL13-, IL-6-, and MMP-13-positive cells in the SCCR2 E3 and full-length SCCR2 groups than Mock group, and less in the sCCR2 E3 group than the full-length sCCR2 group (Fig. 4B). In addition, the effects of sCCR2 E3 on OA were analyzed using mesenchymal stem cells (MSCs) derived from OA patients. MSCs of OA patients were transfected Mock or SCCR2 E3 and then stimulated with lipopolysaccharide (LPS) and MCP-1. The mRNA level of SOX9, a chondrogenic factor, increased whereas those of MMP-1 and MMP-3, catabolic factors, decreased, in the sCCR2 E3 gene group (Fig. 4C). These data indicate that the sCCR2 E3 gene inhibits MCP-1 activity in MSCs by activating chondrogenic factors and inhibiting catabolic factors.

sCCR2 reduces damage in the small intestine. The inflammatory and nociceptive signals from a joint are transmitted to the brain through an afferent arc. The thus affected brain initiates a change in the acetylcholine/epinephrine balance through the efferent arc, which causes inflammation in the intestine. H\&E staining showed that the SCCR2 gene exerted protective effects against intestine damage, and the sCCR2 E3 gene was more effective than full-length sCCR2 (Fig. 5A). Intestinal damage was determined by evaluating epithelial damage and the infiltration of inflammatory cells. The results showed that the sCCR2 E3 gene had better protective effects compared to the full-length sCCR2 gene (Fig. 5B). To confirm the expression of inflammatory cytokines, we performed IHC staining of the small intestine villi (Fig. 6A). The results showed that the number of MCP-1-, CCR2-, and IL-17-positive cells was markedly decreased in the sCCR2 E3 group (Fig. 6B). These results indicate that the SCCR2 E3 gene has protective effects against intestinal damage.

\section{Discussion}

MCP-1/CCL2 is a chemokine that plays an important role in recruiting monocytes/macrophages and recruits monocytes to the site of inflammation through CCR2[5]. Inflammatory responses through the 
CCL2-CCR2 pathway and associated damage cause various diseases such as auto-immune disorders including RA, cancer, atherosclerosis, myocardial infarction, viral infections, and OA.

There are two forms of CCR2, CCR2A and CCR2B, which have structural differences in the C-terminal tails; each form has a different mode of action and mechanism[14]. The expression of CCR2A was increased by CCL2 in an experiment on synoviocytes extracted from RA patients[15]. The binding affinity of CCR2 depends on the extracellular region, with the $\mathrm{N}$-terminal region and E3 domain playing important roles[16-18].

CCR2 has dual functions. Blocking CCR2 leads to improvement in clinical signs and histological scores in the early phase of $O A$ (days 0 to 15) but aggravates clinical and histological signs in the delayed phase (days 21 to 36), the latter which is caused by a humoral immune response involving regulatory $T$ cells[19]. In addition, collagen-induced arthritis in CCR2-null mice exhibited a more severe RA pattern, and it was confirmed that CCR2 also plays a protective role in RA[20]. As such, CCR2 has both pro- and antiinflammatory effects, and special care is required when developing therapeutic drugs that target this protein[8].

Recently, the roles of cytokines and chemokines in OA pathogenesis have garnered increasing attention. Unlike RA, CCL2-CCR2 plays an important role in the progression of OA compared to other chemokine receptors $[4,21]$. Thus, studies on OA treatments that target the CCL2-CCR2 pathway have been increasing. Xu et al. (2015) sampled chondrocytes from OA patients and performed MCP-1 stimulation; they found increased expression of MCP-1, CCR2, and MMP-13, as well as the induction of apoptosis of OA chondrocytes. Based on these studies, various drugs that block CCL2-CCR2 as therapeutic agents for $\mathrm{OA}$ as well as for RA are being developed and tested. As mentioned above, blockade using various molecules such as monoclonal antibodies or soluble receptors of CCL2 and CCR2 in RA has been effective in many animal experiments but has not obtained therapeutic effects in clinical trials[13]. There may be various explanations depending on each experiment, but the redundancy of action between chemokines and chemokine receptors composed of multiple ligands and receptors is likely the major cause[22]. Because of this redundancy, when a single ligand or receptor is targeted, the specificity decreases, leading to ineffective results[23, 24]. Izhak et al.(2009) demonstrated through experiments that a fusion protein comprising as few as 20 amino acids of the E3 domain of the CCL2 receptor increases binding affinity compared to conventional CCR2 antagonists. In addition, in animal experiments, circulating blood levels of CCL2 increased only 1.8-fold after E3-Ig administration, indicating that E3-Ig can act as a more effective therapeutic agent. By contrast, a 2000 -fold increase was observed in other studies using anti-CCL2 monoclonal antibodies [16, 25]. Based on these results, researchers have been able to obtain meaningful results by conducting experiments in which sCCR2 E3 gene therapy was applied to an OA model.

Transfection of the sCCR2 E3 gene into MSCs sampled from an OA patient was followed by treatment with LPS and MCP-1. It was confirmed that SOX9 increased whereas MMP-1 and MMP-3 decreased. This means that the SCCR2 E3 gene induced a chondrogenic effect and inhibited catabolic factors by blocking 
the action of MCP-1 in MSCs. Thus, MCP-1 is increased in OA and induces a pathologic condition, whereas CCR2 inhibits OA pathogenesis by blocking this action of MCP-1.

In OA patients, the suppression of inflammation and the control of pain play very important roles in the quality of life. Fundamentally, treatment with nonsteroidal anti-inflammatory drugs targets inflammation but has an insufficient effect on controlling joint cartilage destruction, so a treatment that simultaneously treats pain and cartilage destruction is urgently needed. In a previous OA surgical model, MCP-1 and CCR2 increased significantly in L3-L5 dorsal root ganglia, which govern pain signals from peripheral joints, and the calcium response in neurons increased significantly upon MCP-1 stimulation. Comparison of the CCR2-null and CCR2-antagonist groups with the wild-type (WT) destabilization of the medial meniscus (DMM) group confirmed that there was a significant decrease in pain and an increase in travel distance. This further confirms that the regulation of MCP-1/CCR2 can help with controlling pain in the peripheral joints[26]. In this study, based on the above results, we attempted to confirm the efficacy of controlling MCP-1 in pain reduction and cartilage protection. To this end, we conducted experiments comparing Mock, full-length sCCR2, and SCCR2 E3 gene therapy in an OA rat model, where MIA was administered to the knee joint to maximize pain and joint destruction. PWT, PWL, and weight-bearing measurements showed that the pain severity decreased, and there was no difference between the groups receiving full-length sCCR2 and SCCR2 E3 gene therapy. Through micro-CT analyses of the three groups, it was confirmed that bone resorption was decreased in the sCCR2 E3 group, with a greater decrease observed in the full-length sCCR2 group. When the knee joint tissues of the three groups were sampled and stained with safranin 0, proteoglycan depletion was less severe in the SCCR2 E3 group compared to the full-length SCCR2 group. The OARSI and Mankin scores were also lower, confirming that cartilage destruction was prevented more effectively. In analyses of the inflammatory cytokines and catabolic factors IL-1 $\beta$, IL-6, and MMP-13 based on IHC staining of samples from the three groups, the SCCR2 E3 group exhibited a greater decrease in levels compared to the full-length SCCR2 group, indicating that sCCR2 E3 had a more effective anti-inflammatory effect. Other previous studies have demonstrated that CCL2-CCR2 is associated with OA and plays a key role in the inflammatory response; however, no clear evidence on its association with chondropathy has been provided. MiotlaZarebska et al. (2017)performed histological analyses and scoring of $\mathrm{CCL}^{-/-}$and $\mathrm{CCR} 2^{-/-}$mice after DMM, but no significant differences with the WT mice were observed [12].Xu et al. (2015) injected a CCR2 antagonist (Sigma) in an MIAinduced OA rat model, but there was no significant improvement based on micro-CT analyses and the pathology score. In this study, we demonstrated through improvement in micro-CT outcomes and OARSI and Mankin scores that SCCR2 E3 gene therapy can prevent cartilage destruction.

In each of the four groups (WT, Mock, sCCR2, full-length sCCR2), the small intestine was sampled at 14 days after treatment administration, and the degree of damage according to the levels of epithelial damage and inflammatory cell infiltration was measured. The least damage was observed in the SCCR2 E3 group. Also, the expression of MCP-1, CCR2, and IL-17 in the intestine was confirmed through IHC staining, with the SCCR2 E3 group exhibiting the greatest inhibition. Through this, we demonstrated that intestinal damage and inflammation were most suppressed via sCCR2 E3 gene therapy targeting the knee 
joint. Regarding the connection between the knee joint and intestine, based on existing studies, the experiment was conducted based on expectations of a connection via the joint-brain-intestine pathway. When joint inflammatory and nociceptive signals are produced, they are transmitted to the brain through an afferent arc, and the brain stimulates the vagus nerve through an efferent arc. Then, acetylcholine is secreted, resulting in a cholinergic anti-inflammatory response [27]. In this process, the afferent arc is stimulated by cytokines secreted from the joint, with CCL2 expected to play a key role. Although the role of CCL2 has not yet been demonstrated, the above speculation can be made based on reports of disruption in the integrity of the blood-brain barrier via the CCL2-CCR2 pathway[28, 29]. When the brain is stimulated by a joint pathology, it is predicted that changes in the intestinal immune environment will occur through changes in hormone homeostasis resulting from alterations in the acetylcholine/epinephrine balance $[28,30]$. In this study, we were able to partially prove the association.

OA has a different immunopathology compared to RA as a low-grade inflammatory disorder, and the CCL2-CCR2 pathway plays a more important role in the former[21]. Therefore, CCL2-CCR2 blockage through sCCR2 E3 is expected to have better therapeutic effects in OA than in RA. In this study, we proved that SCCR2 E3 can block the CCL2-CCR2 pathway more specifically than can other CCR2 antagonists in OA because of its high binding affinity, which solves some of the redundancy problems between multiple ligands and receptors. However, because the inflammatory response within the knee joint is a function of many different multiple pathways, its effect may be limited in actual clinical trials[31]. The redundancy problem will need to be resolved to obtain a meaningful therapeutic effect, and various approaches and studies, such as multiple targeting, as well as a method for increasing the specificity between chemokines and ligands are required.

\section{Abbreviations}

OA: Osteoarthritis

MCP-1: Monocyte chemoattractant protein-1

CCR2: C-C chemokine receptor type 2

MIA: monosodium iodoacetate

IHC: immunohistochemical

IL: interleukin

MMP-13: matrix metalloproteinase 13

CT: Micro-computed tomography

SOX9: SRY-box transcription factor 9

PWL: paw withdrawal latency 
PWT: paw withdrawal threshold

OARSI: Osteoarthritis Research Society International

LPS: lipopolysaccharide

DMM: destabilization of the medial meniscus

\section{Declarations}

\section{Ethics Statement}

This study was carried out in accordance with the recommendations of Ethic Committee of the Catholic University of Korea (CUMC: 2017-0297-01).

\section{Consent for publication}

Not applicable.

\section{Availability of data and materials}

All datasets generated for this study are included in the article.

\section{Conflict of Interests}

The authors have no competing financial interests.

\section{Funding}

This research was supported by the Bio \& Medical Technology Development Program of the National Research Foundation (NRF) funded by the Korean government (MSIP)(NRF-2017M3A9B4028022).

\section{Authors' Contributions}

HSN, SYL, SJK and MLC designed the experiments, analyzed the data. DHL, HSN and JSW wrote the manuscript along with input SJK and MLC. The data performed all in vitro and in vivo assays with HSN, SAK, EJG and ARL. KHC and JWC conducted all immunohistochemistry experiments. All authors critically reviewed and approved the final form of the manuscript.

\section{Acknowledgments}

We thank the Institutional Animal Care and Use Committee, School of Medicine, Catholic University of Korea, for help with animal care and study.

\section{References}


1. Martel-Pelletier J, Barr AJ, Cicuttini FM, Conaghan PG, Cooper C, Goldring MB, Goldring SR, Jones G, Teichtahl AJ, Pelletier JP: Osteoarthritis. Nat Rev Dis Primers 2016, 2:16072.

2. Berenbaum F: Osteoarthritis as an inflammatory disease (osteoarthritis is not osteoarthrosis!). Osteoarthritis and Cartilage 2013, 21:16-21.

3. Haseeb A, Haqqi TM: Immunopathogenesis of osteoarthritis. Clin Immuno/2013, 146:185-196.

4. Wojdasiewicz P, Poniatowski ŁA, Szukiewicz D: The role of inflammatory and anti-inflammatory cytokines in the pathogenesis of osteoarthritis. Mediators of inflammation 2014, 2014.

5. Deshmane SL, Kremlev S, Amini S, Sawaya BE: Monocyte chemoattractant protein-1 (MCP-1): an overview. J Interferon Cytokine Res 2009, 29:313-326.

6. O'Hayre M, Salanga CL, Handel TM, Allen SJ: Chemokines and cancer: migration, intracellular signalling and intercellular communication in the microenvironment. Biochem $J$ 2008, 409:635-649.

7. Harigai M, Hara M, Yoshimura T, Leonard EJ, Inoue K, Kashiwazaki S: Monocyte chemoattractant protein-1 (MCP-1) in inflammatory joint diseases and its involvement in the cytokine network of rheumatoid synovium. Clinical immunology and immunopathology 1993, 69:83-91.

8. Quinones MP, Estrada CA, Kalkonde Y, Ahuja SK, Kuziel WA, Mack M, Ahuja SS: The complex role of the chemokine receptor CCR2 in collagen-induced arthritis: implications for therapeutic targeting of CCR2 in rheumatoid arthritis. $J$ Mol Med (Berl) 2005, 83:672-681.

9. Feria M, Díaz-González F: The CCR2 receptor as a therapeutic target. Expert Opinion on Therapeutic Patents 2006, 16:49-57.

10. Wang J, Seo MJ, Deci MB, Weil BR, Canty JM, Nguyen J: Effect of CCR2 inhibitor-loaded lipid micelles on inflammatory cell migration and cardiac function after myocardial infarction. Int $J$ Nanomedicine 2018, 13:6441-6451.

11. Xu YK, Ke Y, Wang B, Lin JH: The role of MCP-1-CCR2 ligand-receptor axis in chondrocyte degradation and disease progress in knee osteoarthritis. Biol Res 2015, 48:64.

12. Miotla Zarebska J, Chanalaris A, Driscoll C, Burleigh A, Miller RE, Malfait AM, Stott B, Vincent TL: CCL2 and CCR2 regulate pain-related behaviour and early gene expression in post-traumatic murine osteoarthritis but contribute little to chondropathy. Osteoarthritis Cartilage 2017, 25:406-412.

13. Carter PH: Progress in the discovery of CC chemokine receptor 2 antagonists, 2009 - 2012. Expert Opin Ther Pat 2013, 23:549-568.

14. Charo IF, Myers SJ, Herman A, Franci C, Connolly AJ, Coughlin SR: Molecular cloning and functional expression of two monocyte chemoattractant protein 1 receptors reveals alternative splicing of the carboxyl-terminal tails. Proc Natl Acad Sci U S A 1994, 91:2752-2756.

15. Cho M-L, Yoon B-Y, Ju J-H, Jung YO, Jhun J-Y, Park M-K, Park S-H, Cho C-S, Kim H-Y: Expression of CCR2A, an isoform of MCP-1 receptor, is increased by MCP-1, CD40 ligand and TGF- $\beta$ in fibroblast like synoviocytes of patients with RA. Experimental \& molecular medicine 2007, 39:499-507.

16. Izhak L, Wildbaum G, Zohar Y, Anunu R, Klapper L, Elkeles A, Seagal J, Yefenof E, Ayalon-Soffer M, Karin N: A novel recombinant fusion protein encoding a 20 -amino acid residue of the third 
extracellular (E3) domain of CCR2 neutralizes the biological activity of CCL2. J Immuno/ 2009, 183:732-739.

17. Monteclaro FS, Charo IF: The Amino-terminal Domain of CCR2 Is Both Necessary and Sufficient for High Affinity Binding of Monocyte Chemoattractant Protein 1 RECEPTOR ACTIVATION BY A PSEUDOTETHERED LIGAND. Journal of Biological Chemistry 1997, 272:23186-23190.

18. Datta-Mannan A, Stone MJ: Chemokine-binding specificity of soluble chemokine-receptor analogues: identification of interacting elements by chimera complementation. Biochemistry 2004, 43:1460214611.

19. Bruhl H, Cihak J, Schneider MA, Plachy J, Rupp T, Wenzel I, Shakarami M, Milz S, Ellwart JW, Stangassinger $\mathrm{M}$, et al: Dual role of CCR2 during initiation and progression of collagen-induced arthritis: evidence for regulatory activity of CCR2+ T cells. J Immuno/ 2004, 172:890-898.

20. Quinones MP, Ahuja SK, Jimenez F, Schaefer J, Garavito E, Rao A, Chenaux G, Reddick RL, Kuziel WA, Ahuja SS: Experimental arthritis in CC chemokine receptor 2-null mice closely mimics severe human rheumatoid arthritis. J Clin Invest 2004, 113:856-866.

21. Raghu H, Lepus CM, Wang Q, Wong HH, Lingampalli N, Oliviero F, Punzi L, Giori NJ, Goodman SB, Chu CR: CCL2/CCR2, but not CCL5/CCR5, mediates monocyte recruitment, inflammation and cartilage destruction in osteoarthritis. Annals of the rheumatic diseases 2017, 76:914-922.

22. Szekanecz Z, Koch AE: Successes and failures of chemokine-pathway targeting in rheumatoid arthritis. Nat Rev Rheumatol 2016, 12:5-13.

23. Zhao Q: Dual targeting of CCR2 and CCR5: therapeutic potential for immunologic and cardiovascular diseases. J Leukoc Biol 2010, 88:41-55.

24. Gong J-H, Yan R, Waterfield J, Clark-Lewis I: Post-onset inhibition of murine arthritis using combined chemokine antagonist therapy. Rheumatology 2004, 43:39-42.

25. Haringman JJ, Gerlag DM, Smeets TJ, Baeten D, van den Bosch F, Bresnihan B, Breedveld FC, Dinant $\mathrm{HJ}$, Legay F, Gram H, et al: A randomized controlled trial with an anti-CCL2 (anti-monocyte chemotactic protein 1) monoclonal antibody in patients with rheumatoid arthritis. Arthritis Rheum 2006, 54:2387-2392.

26. Miller RE, Tran PB, Das R, Ghoreishi-Haack N, Ren D, Miller RJ, Malfait AM: CCR2 chemokine receptor signaling mediates pain in experimental osteoarthritis. Proc Natl Acad Sci U S A 2012, 109:2060220607.

27. Berenbaum F, Meng QJ: The brain-joint axis in osteoarthritis: nerves, circadian clocks and beyond. Nat Rev Rheumatol 2016, 12:508-516.

28. Cox MA, Bassi C, Saunders ME, Nechanitzky R, Morgado-Palacin I, Zheng C, Mak TW: Beyond neurotransmission: acetylcholine in immunity and inflammation. $J$ Intern Med 2020, 287:120-133.

29. Yao Y, Tsirka SE: Monocyte chemoattractant protein-1 and the blood-brain barrier. Cell Mol Life Sci 2014, 71:683-697.

30. Matteoli G, Boeckxstaens GE: The vagal innervation of the gut and immune homeostasis. Gut 2013, 62:1214-1222. 
31. Lebre MC, Vergunst CE, Choi IY, Aarrass S, Oliveira AS, Wyant T, Horuk R, Reedquist KA, Tak PP: Why CCR2 and CCR5 blockade failed and why CCR1 blockade might still be effective in the treatment of rheumatoid arthritis. PLoS One 2011, 6:e21772.

\section{Figures}

A

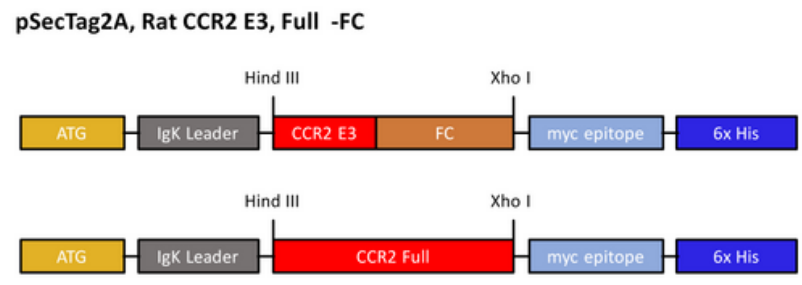

B

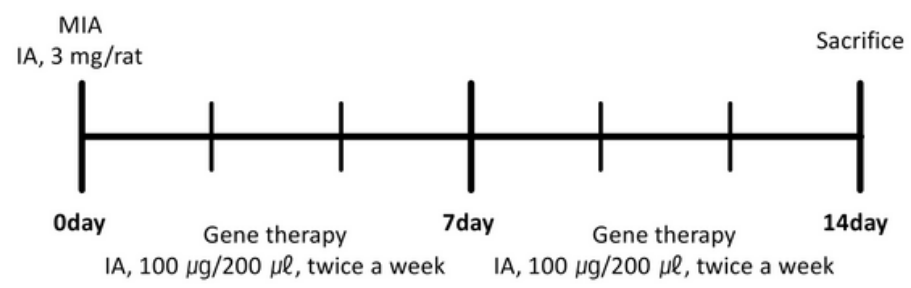

C

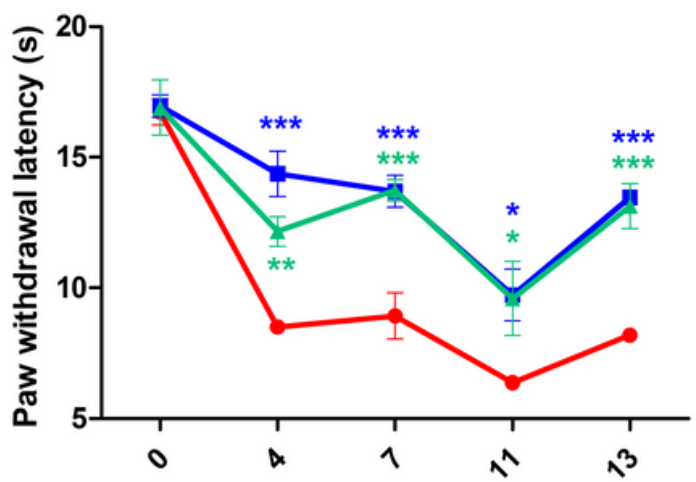

The days after induction of Arthritis

D

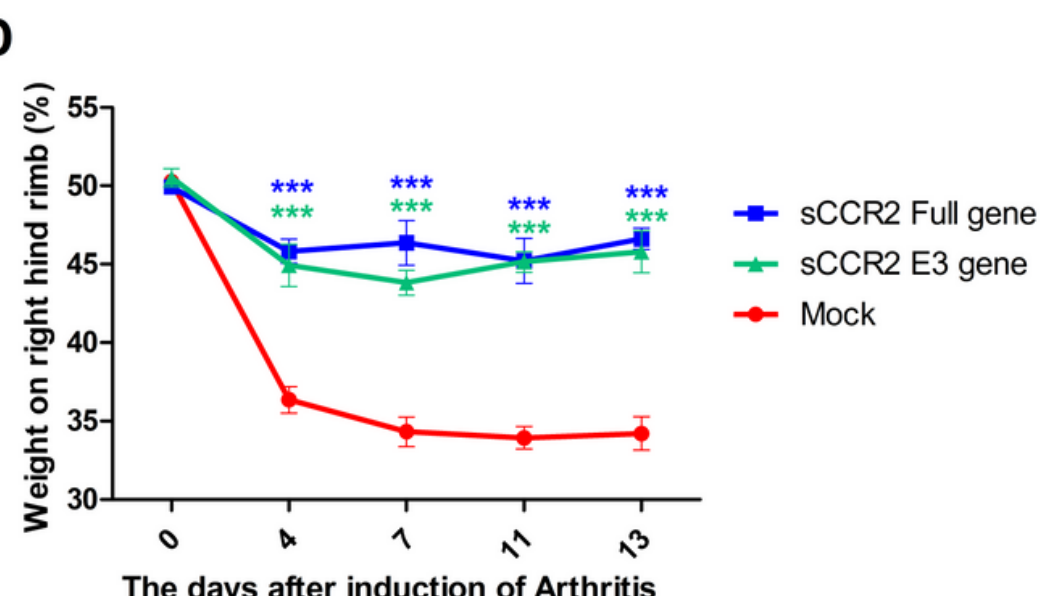

The days after induction of Arthritis

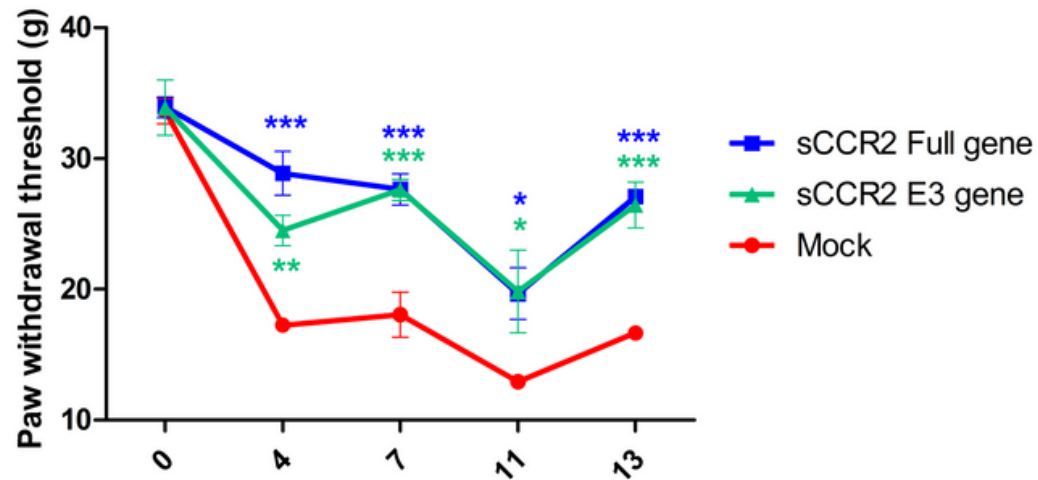

The days after induction of Arthritis

\section{Figure 1}

SCCR2 Regulates the pain threshold in MIA rat (A) A schematic diagram of sCCR2 full and SCCR2 E3 vector map. (B) A schematic diagram of the experimental protocols used demonstrating sCCR2 full and sCCR2 E3 gene treatment in OA $(n=3)$. (C) Nociceptive testing was performed using a dynamic plantar 
esthesiometer (Ugo Basile, Gemonio, Italy), an automated version of the von Frey hair assessment procedure (D) Weight bearing was performed using a incapacitance meter (IITC life science, USA). The data are representative of at least three independent experiments. The data are reported as the mean \pm SEM of three independent experiments. $\left({ }^{*} p<0.05,{ }^{*} p<0.01,{ }^{* \star *} p<0.001\right)$

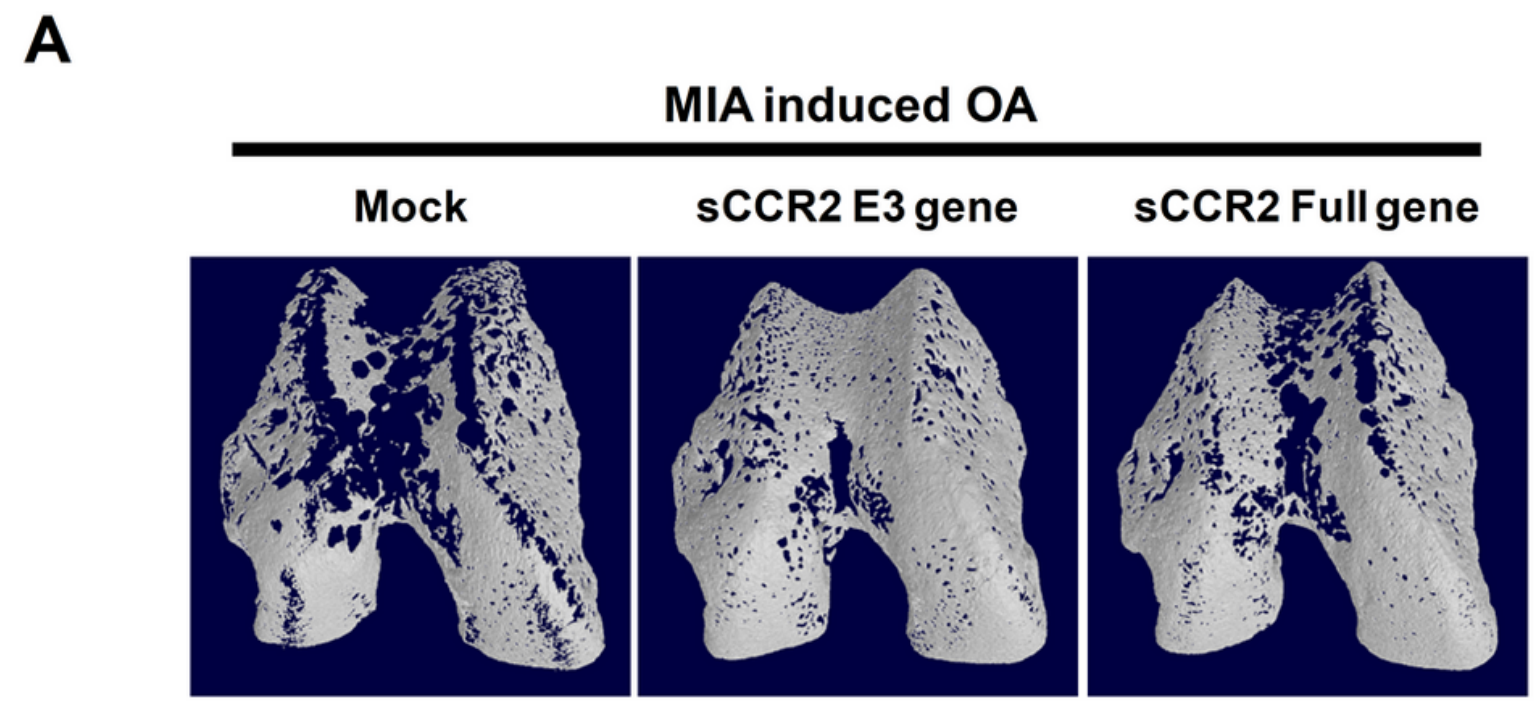

B
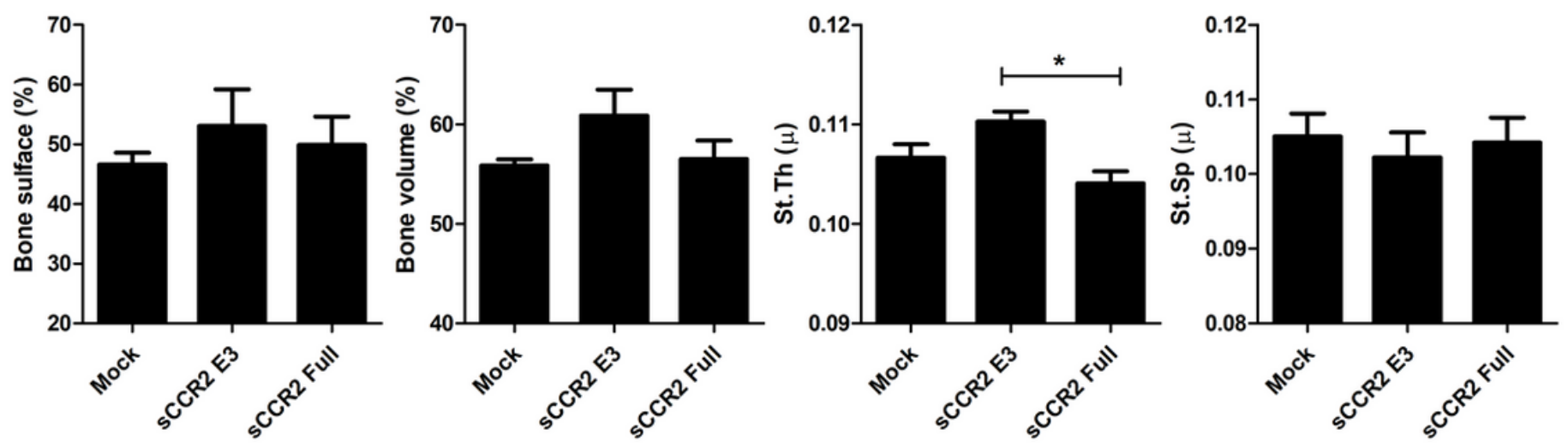

Figure 2

SCCR2 reduces bone resorption in MIA rat (A) A bone biopsy trephine bur ( $3310 \mathrm{~mm}$ ) was used to remove 20 cylindrical bone samples from 20 dry hemimandibles. Samples were scanned using mCT (mCT 35; SCANCO Medical, Br" uttisellen, Switzerland) (B) Bone volume, bone surface; st.th and st.sp were measured to the BoneJ. The data are reported as the mean \pm SEM of three independent experiments. ( ${ }^{*} p<$ 0.05) 
A

\section{MIA induced OA}

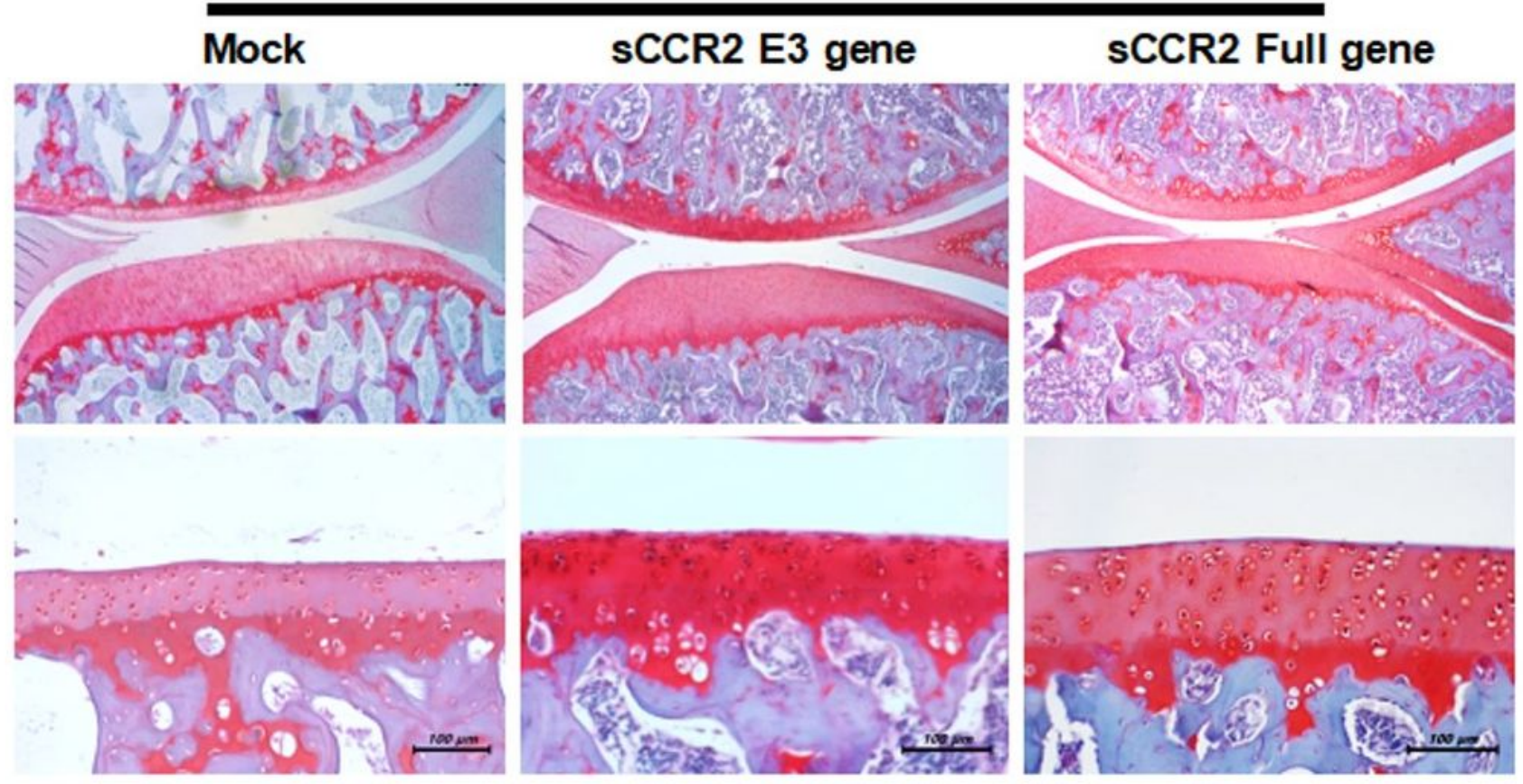

B
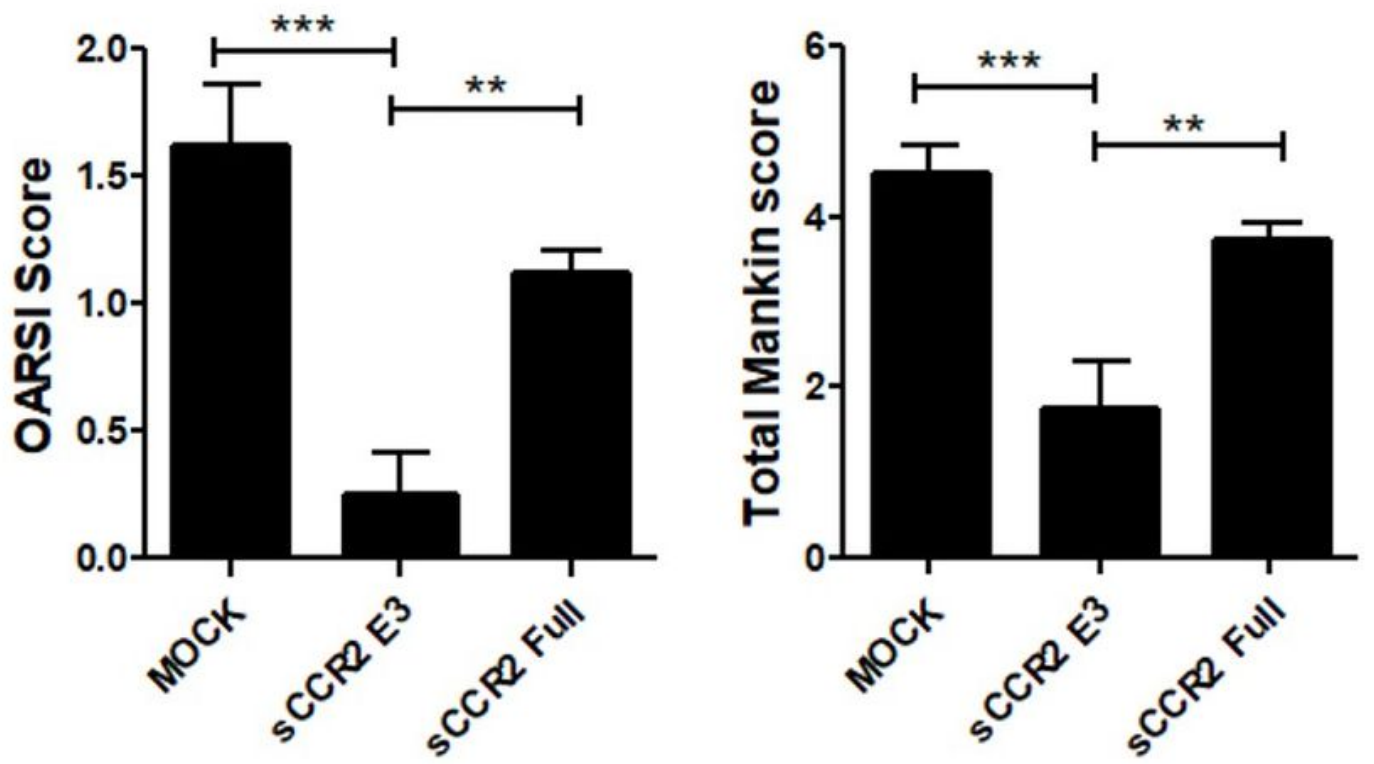

Figure 3

SCCR2 reduces depletion of proteoglycan in MIA rat (A) Knee Joint tissue samples were collected from Mock or SCCR2 E3, sCCR2 full gene rat on 14 day and subjected to stain with safranin O. (B) stained tissues were assesed by Osteoarthritis Research Society International (OARSI) and Mankin scores. The data are reported as the mean \pm SEM of three independent experiments. $\left(\star \star p<0.01,{ }^{\star \star \star} p<0.001\right)$ 

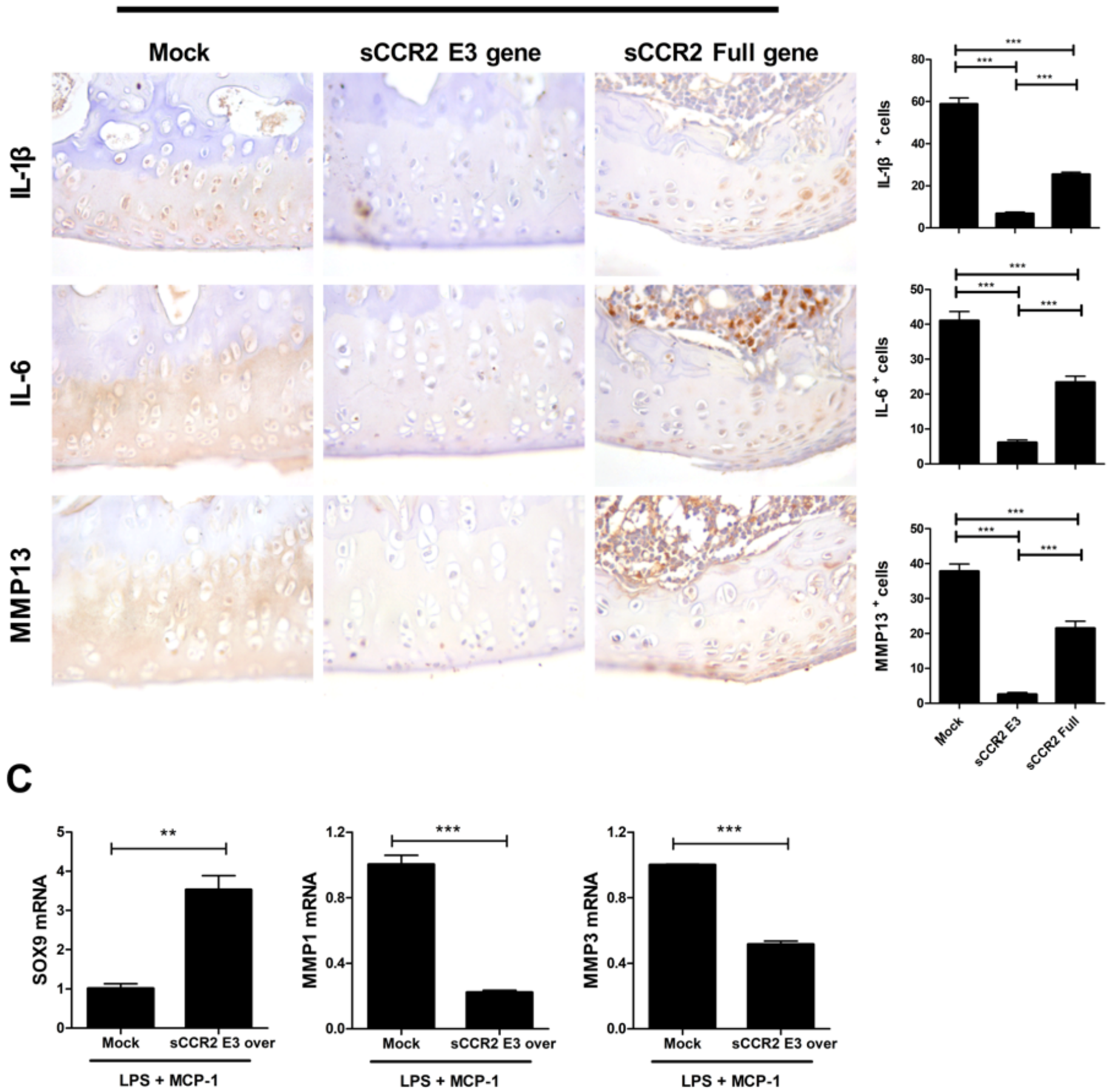

Figure 4

sCCR2 reduces inflammatory cytokines in knee joint (A) Knee joint tissue samples from all group was stained immunohistochemically with specific antibodies to IL-1 $\beta$, IL-6 and MMP13. (B) Immunohistochemically positive cells were counted. (C) MSCs of OA patients were transfected Mock or sCCR2 E3 and then stimulated with LPS $100 \mathrm{ng} / \mathrm{mL}$ and MCP-1 $10 \mathrm{ng} / \mathrm{mL}$. The mRNA level of SOX9, MMP1 and MMP3 were determined using real-time. The data are reported as the mean \pm SEM of three independent experiments. $\left({ }^{*} \mathrm{p}<0.01,{ }^{* \star *} \mathrm{p}<0.001\right)$ 

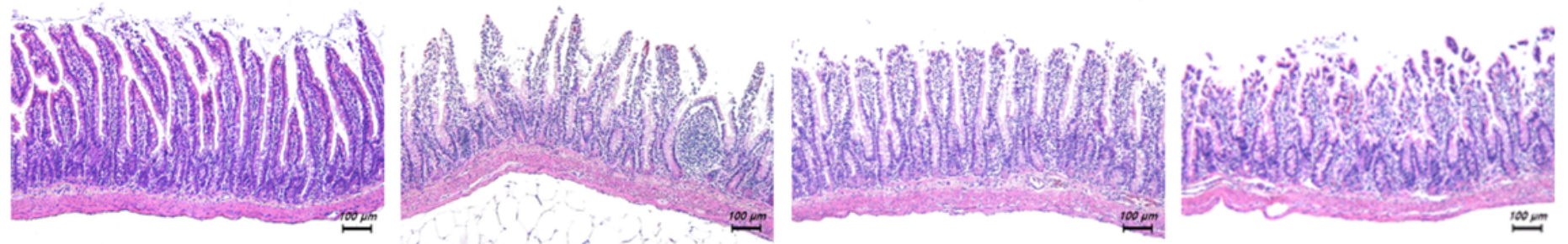

B
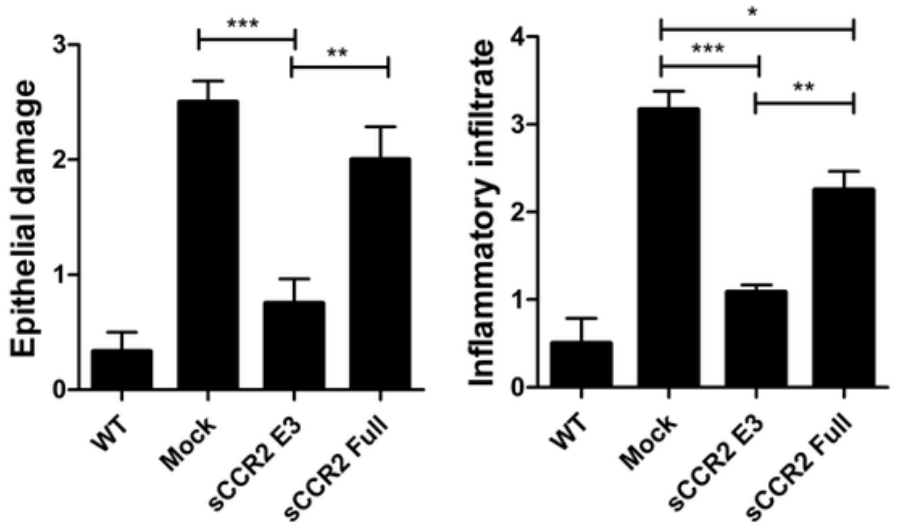

Figure 5

SCCR2 reduces damage in small intestine (A) Small intestine tissue samples were acquired from Mock or SCCR2 E3, sCCR2 full gene rat on 14 day and subjected to stain with H\&E (B) Epithelial and Inflammatory infiltrate scoring. The data are reported as the mean \pm SEM of three independent experiments. $\left({ }^{*} p<0.05\right.$, $\left.{ }^{* \star} p<0.01, * \star * p<0.001\right)$ 原

\section{Figure 6}

sCCR2 reduces inflammation in small intestine (A) Small intestine tissue samples from all group was immunohistochemically stained with specific antibodies to IL-17, MCP-1, CCR2 (B) Immunohistochemically positive cells were counted. The data are reported as the mean \pm SEM of three independent experiments. $\left({ }^{*} p<0.05,{ }^{*} p<0.01,{ }^{* \star *} p<0.001\right)$ 